\title{
Specific-cue effects of interpolated movements on distance and location retention in short-term motor memory
}

\author{
JOSEPH D. HAGMAN \\ New Mexico State University, Las Cruces, New Mexico 88003
}

\begin{abstract}
A short-term motor retention paradigm was used to examine the effect of interpolated movements on the recall of individual distance- and location-cue information. Subjects were instructed to learn either a distance or a location cue during the execution of simple, discrete original movements. Interpolated movements were then made which varied and repeated distance and location either individually or jointly. Changes in variable, absolute, and algebraic error from immediate to delayed recall were totally determined by interpolation of the individ. ual instructed cue. No added recall changes were produced by interpolation of noninstructed cues. The data were interpreted as supporting the presence of a stimulus-selection process that dissociates kinesthetic information as a function of instructional set. The effects of interpolated movements, then, are determined partially by this selection process.
\end{abstract}

Short-term motor memory (STMM) studies have identified distance and location as two sources of kinesthetic information present at the execution of a simple, discrete movement (e.g., Laabs, 1973). Distance refers to movement length, while location refers to movement stopping position.

Recent research efforts have been directed toward discovering the effects of interpolated movements designed to interfere with the retention of individual distance and location cues. In general, interfering interpolated movements have been found to produce response biasing (e.g., Laabs, 1974), increases in withinsubjects variability (e.g., Stelmach \& Walsh, 1973), and decreases in absolute accuracy (e.g., Zahorik, 1972). Response biasing is indicated when recall of an original movement shifts in the direction of the interpolated movement and is revealed when constant error (CE) is measured. Variable error (VE) indicates within-subjects variability and is revealed when the standard deviation of $\mathrm{CE}$ is analyzed. Absolute error (AE) is an indicant of overall response accuracy and is determined by analysis of $\mathrm{CE}$ irrespective of sign.

There have been generally two problems associated with studies designed to examine the effects of interpolated movements on individual distance- and location-cue retention. First, distance and location

This research represents a portion of a dissertation submitted in partial fulfillment of the requirements for the $\mathrm{PhD}$ degree at New Mexico State University under the supervision of Gordon B. Harding. It was presented at the meeting of the Midwestern Psychological Association held in Chicago, May 1977. The author wishes to thank Evelyn Williams for her helpful suggestions and Vi Hagman for her clerical assistance. Requests for reprints should be sent to Joseph D. Hagman, Army Research Institute, 5001 Eisenhower Avenue, Alexandria, Virginia 22333. cues have been confounded at interpolation. That is, both cues have been either varied (i.e., changed relative to their original movement value) or repeated (i.e., not changed relative to their original movement value) during interpolated movements (e.g., Stelmach \& Walsh, 1973). This confounding renders isolation of the facilitation- or interference-causing dimension impossible. Second, such studies (e.g., Laabs, 1974; Zahorik, 1972) typically do not measure immediate recall. This prevents an accurate evaluation of the amount forgotten and makes it difficult to claim that retention is actually being examined.

The result of distance- and location-cue confounding at interpolation is that two hypotheses concerning the retention of individual movement cues cannot be differentiated. One will be called the multiple-cue hypothesis, while the other will be called the specific-cue hypothesis. The multiple-cue hypothesis states that the memorial representation of a movement is derived from many sources of information (Adams, 1972; Diewert, 1975; Stelmach, 1973) (e.g., distance and location), and that retention will be a function of the number of these sources manipulated at interpolation. The specific-cue hypothesis states that the memorial representation of a movement is based upon an individual cue (Adams \& Goetz, 1973; Posner, Nissen, \& Klein, 1976; Russell, 1976) (e.g., distance or location), which is selected for processing during original movement execution. Retention of the processed cue will depend upon whether or not it is manipulated during interpolation.

As support for the multiple-cue hypothesis, Hagman and Williams (1977) have reported that interference from interpolated movements was based upon the number of kinesthetic cues varied at interpolation. However, they allowed subjects to process multiple 
cues at original movement execution. Thus, the applicability of the multiple-cue hypothesis to situations where subjects are requested to process individual original movement cues remains unknown. In support of the specific-cue hypothesis, Zahorik (1972) has shown that variation of individual distance or location cues at interpolation in an individual-cue learning situation is capable of producing retention decrements. However, her results do not differentiate between the multiple- and specific-cue hypotheses because multiple cues were not interpolated.

The present experiment directly compares the predictions of the multiple- and specific-cue hypotheses in a context where learning instructions are used to bias subjects toward the learning of individual movement cues. Although learning instructions have been shown to bias the encoding of movement in favor of an instructed cue (Hagman \& Francis, 1975), it is not clear whether subjects are able to isolate an instructed cue from other uninstructed cues and rely totally upon the former for accurate recall. In addition to using individual-cue learning instructions, it was necessary to eliminate the confounding of distance and location at interpolation and at the same time provide data concerning multiple-cue interpolation effects. This was accomplished by varying and repeating distance and location cues either individually or jointly at interpolation. The purpose of cue variation was to discover the specificity of interference, while cue repetition was employed to determine the specificity of practice on individual-cue retention. In addition, both immediate and delayed recall scores were taken to evaluate the amount forgotten cue to interpolation.

The multiple-cue hypothesis predicts that manipulation of both distance and location at interpolation should produce greater dependent variable changes than individual-cue manipulation. The specific-cue hypothesis predicts that individual-cue manipulation will be sufficient to cause dependent variable changes. These changes should be identical to those produced by multiple-cue manipulation when the cue manipulated at interpolation corresponds to the instructed cue encoded at original movement execution. Thus, under the specific-cue hypothesis, learning instructions should determine which types of interpolated movements will produce dependent variable changes from immediate to delayed recall. The multiple-cue hypothesis, however, does not make this instructional distinction.

\section{METHOD}

\section{Subjects}

One-hundred-and-fifty students participated in the experiment to partially fulfill an introductory psychology course requirement at New Mexico State University.

\footnotetext{
Apparatus

Movements were executed from left to right with a single, freely movable, wooden element. The clement slid horizontally
}

along a wooden bar 34 in. $(87.72 \mathrm{~cm})$ in length and $12 \mathrm{in.}$ $(30.96 \mathrm{~cm}$ ) above a table top. The side of the bar facing the experimenter contained a number of holes into which a stylus was inserted by the experimenter to stop the movements. A chin rest was used to provide control of head movements and body position. Earphones were used to eliminate any auditory cues resulting from movements.

\section{Design}

A 2 by 2 by 6 by 2 by 2 by 5 mixed-treatment design was used. The within-subjects variables were: original movement length, that is, 6 in. $(15.24 \mathrm{~cm})$ and 18 in. $(45.72 \mathrm{~cm})$; deviation of interpolated variation $( \pm 24 \%$ of the original movement); replications (1-6); and time of recall (immediate and delayed). Each student performed a total of 24 original movements formed from the product of two original movement lengths, two interpolation deviations, and six replications. Each original movement was recalled twice; that is, once immediately after execution and once after completion of the retention interval Original movements were randomly presented with each third of the students receiving a different random sequence.

The between-subjects variables were cue instructed (distance and location) and interpolated activity; that is, distance variation and location repetition (DV-LR), distance repetition and location variation (DR-LV), distance and location variation (DV-LV), distance and location repetition (DR-LR), and rest. Fifteen students were randomly assigned to each of 10 conditions formed by the factorial combination of these two between-subjects variables and their associated levels.

The DV-LR students terminated at the same location as the original movement during interpolation (i.e., repeated location), but traveled $\pm 25 \%$ of its distance (i.e., varied distance). The DR-LV students performed interpolated movements of identical distance as the original movement but stopped at locations $\pm 25 \%$ of its location. Students in the DV-LV condition varied both distance and location $\pm 25 \%$ at interpolation. The DR-LR students repeated the original movement distance and location during interpolation, while the rest students did not execute any overt movements on the apparatus during the retention interval. The rest groups were inserted to assess the effects of time alone on individual distance and location retention.

In order to manipulate individual distance and location cues, the starting position was changed at interpdlation. When both distance and location were varied or repeated, the interpolated movement starting position remained indentical to that of the original movement. In all conditions except rest, two identical interpolated movments were always executed.

Both distance and location were always available upon original movement execution, but only individual cues were requested at immediate and delayed recall. Separation of distance and location cues at recall was accomplished by varying the recall starting positions \pm 3 in. $(7.62 \mathrm{~cm})$ in relation to the starting position of the original movement. This type of manipulation has been used previously by others (e.g., Hagman \& Francis, 1975; Laabs, 1973, 1974).

\section{Procedure}

On all trials, the interpolated movement students heard the following tape-recorded sequence of commands over earphones: "ready," "Movement 1," "ready," "recall Movement 1," "ready," "Movement A," "ready," "Movement B," "ready," "recall Movement 1." The rest students heard all the above commands except for those associated with the interpolated movements (Movements $\mathrm{A}$ and $\mathrm{B}$ ). The retention interval, ineasured from completion of initial recall. was $20 \mathrm{sec}$.

At the initial "ready" command, the experimenter grasped the right hand of the student and placed it on the movable slide. Upon hearing "Movement !." the sudent moved the slide at a steady speed from left to reshe until it cance in contact with 
a stop. Then he released his hand from the slide and placed it in front of his body in a standard position on the table. The experimenter then repositioned the slide at a different starting position in preparation for the immediate recall trial. At the next "ready" command, the experimenter placed the right hand of the student on the slide, after which the student recalled the movement upon hearing the "recall" command. Then, as after all movements, the student returned his hand to the standard position on the table in front of his body. The same sequence of commands, excluding the commands associated with immediate recall, occurred for the two subsequent interpolated movements. The two interpolated movements were always designated by the letters $A$ and $B$, while original movements were always designated by numbers. After completing the interpolated movements, recall of the original movement was atternpted. Once Movement 1 was recalled for the second time (delayed recall), the next original movement (Movement 2) was executed, and the sequence continued until all 24 original movements had been executed and recalled. Recall accuracy was measured to the nearest $1 / 8 \mathrm{in} .(3.175 \mathrm{~mm})$ and then was converted into millimeters.

A total of $5 \mathrm{sec}$ was provided for the execution of movements. A period of 3 sec elapsed between each "ready" command and the subsequent command. The interval occurring after final recall and prior to the initial "ready" command of the next trial was $10 \mathrm{sec}$.

\section{Instructions}

Before beginning the experiment, all students were informed of both the distinction between distance and location cues and the type of procedure used to examine their retention. During this preliminary briefing period, the experimenter performed a demonstration on the apparatus. The actual learning instructions directed students to pay attention to and remember either the distance or the location of the original movement, depending upon which cue they were to recall. Students were told that no interpolated movements would have to be recalled. If there were no questions, the students were blindfolded and the experiment began.

\section{RESULTS}

\section{Variable and Absolute Error}

Both VE and mean AE were calculated for individual students over the six replications associated with each of the four original movement conditions. These four conditions were originally formed by the factorial combination of two original movement lengths and two interpolated movement deviations. An analysis of variance was then performed on these scores.

The data were found to be orderly. In general, interpolated movements that varied the instructed cue produced increases from immediate to delayed recall for both VE and AE. Interpolations that repeated the instructed cue produced decreases in VE and $\mathrm{AE}$ from immediate to delayed recall. The additional variation or repetition of noninstructed cues had no augmenting effect on the changes in $\mathrm{VE}$ or $\mathrm{AE}$ produced by manipulation of the instructed cue alone.

Statistical support for the above conclusions was provided by a significant Instructed Cue by Interpolated Activity by Time of Recall interaction for both VE $[F(4,140)=11.62, p<.05]$ and $\operatorname{AE}[F(4,140)=10.02$, $p<.05]$, as summarized in Table 1. Based upon this significant higher order interaction, the least squared difference test (LSD) was used to examine simple effects (Carmer \& Swanson, 1973). The rejection region for all individual comparisons was .05 .

When distance was the instructed cue, significant increases in VE from immediate to delayed recall occurred for the DV-LR and DV-LV interpolation conditions $[\operatorname{LSD}(140)=6.92$ and $\operatorname{LSD}(140)=6.76$, respectively]. Significant increases in $\mathrm{AE}$ were also found for the DV-LR $[\operatorname{LSD}(140)=4.67]$ and DV-LV $[\operatorname{LSD}(140)=6.08]$ conditions. Nonsignificant $t$ values resulting from the comparison of individual student difference scores (delayed minus immediate recall) indicated that the two interpolation conditions did not show differential VE or AE increases. Thus, variation of the additional noninstructed location cue during interpolation had no effect on recall over and above the effect produced by variation of the instructed distance cue alone. Significant decreases in VE from immediate to delayed recall were found for the DR-LV [LSD $(140)=$ -3.88] and DR-LR conditions [LSD $(140)=-6.70]$. Decreases in AE were also found for the DR-LV and DRLR conditions $[\operatorname{LSD}(140)=-4.16$ and -5.78 , respectively]. No differences were found in the amount of VE

Table 1

Mean Variable and Absolute Error (in millimeters) for Immediate and Delayed Recall of Distance and Location as a Function of In terpolated Activity

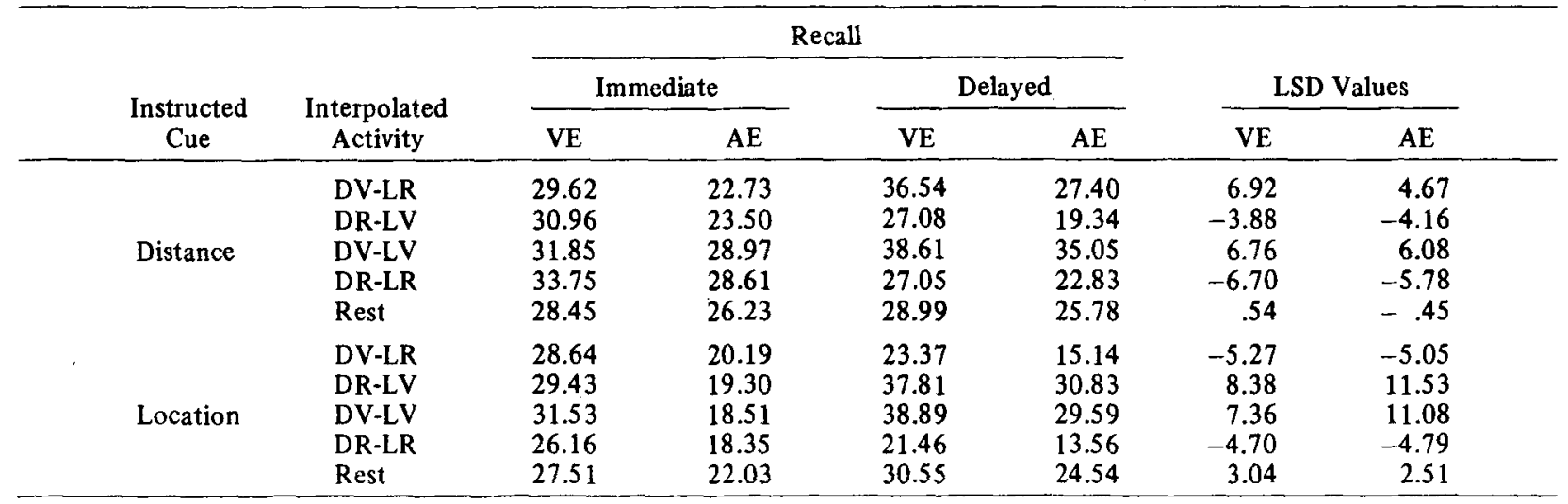


or $\mathrm{AE}$ decreases produced by these two interpolation conditions as indicated by nonsignificant differencescore $t$ values. Thus, repetition of the noninstructed location cue had no additional effect on either dependent variable relative to the decreases produced by repetition of the individual distance cue.

When location was the instructed cue, significant increases in VE from immediate to delayed recall occurred for the DR-LV [LSD $(140)=8.38]$ and DV-LV $[\operatorname{LSD}(140)=7.36]$ conditions. The same was true for $\mathrm{AE}[\operatorname{LSD}(140)=11.53$, for the DR-LV condition; $\operatorname{LSD}(140)=11.08$, for the DV-LV condition]. The difference-score $t$ values were nonsignificant, indicating that the degree of recall decrement did not differ between the two interpolation conditions for either VE or AE. Thus, no effect of varying the noninstructed distance cue in the DV-LV condition was evident. Significant decreases in VE were found for the DV-LR [LSD $(140)=-5.27]$ and DR-LR conditions $[\operatorname{LSD}(140)=-4.70]$. The $\mathrm{AE}$ scores revealed similar recall facilitation caused by location repetition in the DV-LR [LSD(140)=-5.05] and DR-LR $[\operatorname{LSD}(140)=-4.79]$ conditions. Once again, the difference-score $t$ values were nonsignificant, indicating the VE and AE decreases were not affected by repetition of distance in the DR-LR condition.

The distance- and location-cue rest conditions exhibited no significant changes in $\mathrm{VE}$ or $\mathrm{AE}$ from immediate to delayed recall. Finally, in agreement with previous research (e.g., Stelmach, 1970), the main effect of original movement length was found to be significant for both VE $[F(1,140)=116.51, p<.05]$ and $A E$ $[F(1,140)=36.61, p<.05]$, revealing greater error associated with the long original movement than with the short original movement.

\section{Constant Error}

Mean $\mathrm{CE}$ was calculated for individual students from the same scores used to obtain $\mathrm{VE}$ and mean $\mathrm{AE}$ estimates. An analysis of variance was then performed on these means. In general, the interpolated movements that produced $\mathrm{VE}$ and $\mathrm{AE}$ changes from immediate to delayed recall also produced $\mathrm{CE}$ shifts in their specific direction. Statistical support for the above statement was provided by a significant Instructed Cue by Interpolated Activity by Interpolated Deviation by Time of Recall interaction $[\mathrm{F}(4,140)=11.05, \mathrm{p}<.05]$, summarized in Table 2. Again, the simple effects were tested using the least squared difference test, with the rejection region equal to .05 for all individual comparisons.

When location was the instructed cue, significant positive (in the direction of greater extent) error shifts occurred from immediate to delayed recall in the DR-LV $[\operatorname{LSD}(140)=13.17]$ and DV-LV conditions $[\operatorname{LSD}(140)=15.35]$. Significant negative error shifts (in the direction of lesser extent) were produced by the DR-LV [LSD $(140)=-17.78]$ and DV-LV interpolation conditions $\quad[\operatorname{LSD}(140)=-16.67]$. Difference-score $t$ values for these four conditions showed no differential $\mathrm{CE}$ shifts between either the two positive or two negative shift conditions. Thus, additional variation of noninstructed cues added nothing to the error shifts already produced by variation of individual instructed cues. The conditions in which location was repeated (i.e., DR-LR and DV-LR) showed a slight nonsignificant increase in accuracy from immediate to delayed recall. Apparently, the effect of repetition shows up better in lessened VE and $\mathrm{AE}$ than in shifted CE. To avoid misinterpretation, it should be realized that in Table 2 the positive and negative interpolation designations associated with the DR-LR and rest conditions were inserted for purposes of statistical analysis. Actually, subjects in the positive and negative DR-LR conditions always repeated the original movement, while those in the rest conditions did not perform any interpolated movements on the apparatus.

When distance was the instructed cue, significant

Table 2

Mean Constant Error (in millimeters) for Immediate and Delayed Recall of Distance and Location as a Function of the Direction of Interpolated Activity

\begin{tabular}{|c|c|c|c|c|c|c|c|}
\hline \multirow{3}{*}{$\begin{array}{c}\text { Interpolated } \\
\text { Activity }\end{array}$} & \multirow{3}{*}{$\begin{array}{c}\text { Direction of } \\
\text { Interpolation }\end{array}$} & \multicolumn{3}{|c|}{ Location Instruction } & \multicolumn{3}{|c|}{ Distance Instruction } \\
\hline & & \multicolumn{2}{|c|}{ Time of Recall } & \multirow{2}{*}{$\begin{array}{c}\text { LSD } \\
\text { Values }\end{array}$} & \multicolumn{2}{|c|}{ Time of Recall } & \multirow{2}{*}{$\begin{array}{l}\text { LSD } \\
\text { Values }\end{array}$} \\
\hline & & Immediate & Delayed & & Immediate & Delayed & \\
\hline DV-LR & $\begin{array}{l}\text { Positive } \\
\text { Negative }\end{array}$ & $\begin{array}{r}4.29 \\
.62\end{array}$ & $\begin{array}{r}1.14 \\
-\quad .07\end{array}$ & $\begin{array}{r}-3.15 \\
-\quad .69\end{array}$ & $\begin{array}{r}5.43 \\
-5.05\end{array}$ & $\begin{array}{r}18.63 \\
-12.41\end{array}$ & $\begin{array}{r}13.20 \\
-7.36\end{array}$ \\
\hline DR-LV & $\begin{array}{l}\text { Positive } \\
\text { Negative }\end{array}$ & $\begin{array}{l}-7.52 \\
-5.84\end{array}$ & $\begin{array}{r}5.65 \\
-23.62\end{array}$ & $\begin{array}{r}13.17 \\
-17.78\end{array}$ & $\begin{array}{l}-5.30 \\
-6.54\end{array}$ & $\begin{array}{r}5.72 \\
-4.35\end{array}$ & $\begin{array}{r}11.02 \\
2.19\end{array}$ \\
\hline DV-LV & $\begin{array}{l}\text { Positive } \\
\text { Negative }\end{array}$ & $\begin{array}{r}.89 \\
-4.67\end{array}$ & $\begin{array}{r}16.24 \\
-21.34\end{array}$ & $\begin{array}{r}15.35 \\
-16.67\end{array}$ & $\begin{array}{l}-11.49 \\
-16.38\end{array}$ & $\begin{array}{r}1.97 \\
-25.08\end{array}$ & $\begin{array}{r}13.46 \\
-8.70\end{array}$ \\
\hline DR-LR & $\begin{array}{l}\text { Positive } \\
\text { Negative }\end{array}$ & $\begin{array}{r}6.06 \\
10.80\end{array}$ & $\begin{array}{l}3.75 \\
6.51\end{array}$ & $\begin{array}{l}-2.31 \\
-\quad 4.29\end{array}$ & $\begin{array}{r}-11.14 \\
-6.25\end{array}$ & $\begin{array}{l}-3.97 \\
-1.59\end{array}$ & $\begin{array}{l}7.17 \\
4.66\end{array}$ \\
\hline Rest & $\begin{array}{l}\text { Positive } \\
\text { Negative }\end{array}$ & $\begin{array}{l}4.83 \\
6.16\end{array}$ & $\begin{array}{r}.83 \\
7.21 \\
\end{array}$ & $\begin{array}{r}-4.00 \\
1.05 \\
\end{array}$ & $\begin{array}{r}-5.30 \\
-\quad 3.33 \\
\end{array}$ & $\begin{array}{l}-2.67 \\
-\quad 4.73 \\
\end{array}$ & $\begin{array}{r}2.63 \\
-1.40\end{array}$ \\
\hline
\end{tabular}


positive error shifts from immediate to delayed recall occurred for positive interpolated variation in the DV.LR $[\operatorname{LSD}(140)=13.2]$ and DV-LV conditions $[\operatorname{LSD}(140)=13.46]$. Negative interpolation was found to cause negative $\mathrm{CE}$ shifts in the same two conditions $[\operatorname{LSD}(140)=-7.36$, and $\operatorname{LSD}(140)=-8.70$, respectively]. Difference-score $t$ values did not reveal differential CE shifts between these two conditions after either positive or negative interpolation. The addition of a noninstructed location cue during variation of the instructed distance cue contributed no added CE shifts over and above those produced by the instructed cue.

Interpolation of negative location in the DR-LV condition had no effect on CE shifts, while positive location interpolation in the same condition had a significant response-biasing effect $[\operatorname{LSD}(140)=11.02]$. In this particular case, location variation affected recall of distance. Speculation as to why this effect occurred will not be attempted because it is considered spurious for two reasons. First, negative location interpolation did not produce negative CE shifts. Thus, negative and positive interpolation did not have the same effect. Second, the effect of positive interpolation was inconsistent with the pattern of results produced by previous conditions including the negative location condition. Visual inspection of Table 2 reveals a slight increase in accuracy for both DR-LR conditions and the DR-LV negative condition. The increases were statistically supported in only the DR-LR positive condition $[\operatorname{LSD}(140)=7.17]$. Perhaps the other two conditions suffered from a ceiling effect caused by very accurate immediate recall.

Table 2 reveals that changes in $\mathrm{CE}$ from immediate to delayed recall were not apparent for the distanceor location-cue rest conditions. Unfilled retention intervals, therefore, had no effect on the recall of either cue.

A number of additional results were found that are consistent with earlier findings. The main effect of original movement length was significant $[F(1,140)=43.32, p<.05]$ and revealed a central tendency effect (also called the range effect) where the short original movement was overshot and the long original movement was undershot at recall (e.g., Hagman \& Williams, 1977). Although this central tendency effect was present for both distance and location recall, it was more pronounced with distance as evidenced by the significant Criterion Length by Cue Recalled interaction $[F(1,140)=18.42, p<.05]$. And finally, a significant main effect of interpolated movement direction $[F(1,140)=30.68, p<.05]$ showed that negative interpolations caused recall to shift in a negative direction, while positive interpolation shifted recall in a positive direction as reported previously (e.g., Craft \& Hinrichs, 1971).

\section{DISCUSSION}

The data support the specific-cue hypothesis in that the effects of interpolated movements depended upon the specific kinesthetic cues manipulated during the retention interval when individual-cue learning instructions were administered. Interpolated variation of the instructed cue resulted in significant increases in $\mathrm{VE}$ and $\mathrm{AE}$ in addition to significant $\mathrm{CE}$ shifts in the direction of the interpolated movements. Interpolated repetition of the instructed cue produced consistent decreases in VE and $A E$. Neither repetition nor variation of noninstructed kinesthetic cues had a supplemental effect on recall relative to the effect produced by manipulation of the instructed cue alone.

The specificity with which interpolated movements affected all three dependent variables implies that a cognitive process such as stimulus selection was active during the course of a retention trial. Although stimulus selection has been investigated for years in the area of verbal learning (e.g., Underwood, 1963), it has only recently been suggested to occur in motor learning (Adams \& Goetz, 1973; Gundry, 1975; Hagman \& Francis, 1975). In the present study, it is argued that students selected a single kinesthetic cue from among multiple kinesthetic cues with the intention of maximizing recall of the selected cue. Learning instructions had the effect of guiding this cue-selection process.

It is difficult to determine the nature and locus of the selection process; however, some suggestions will be made. Traditionally, attentional mechanisms have been recruited to explain asymmetrical cue selection. For example, Adams and Goetz (1973) have invoked differential attention to account for selection of visual cues over kinesthetic cues in a motor learning task. The locus of differential attention may be at initial encoding, as suggested by Hagman and Francis (1975), or it may occur at the time of rehearsal. Posner (1973) has reported evidence supporting the general notion of a cue-specific rehearsal process. In addition, the ability of students to selectively rehearse specific cues as a function of recall expectancies has been shown to occur with other types of multicodable stimuli, such as pictures (e.g., Frost, 1972). Perhaps the same process occurs with multicodable kinesthetic stimuli (Wrisberg, 1975). The validity of a differential rehearsal argument to explain specific-cue effects is contingent upon the rehearsability of individual kinesthetic cues. The results of the rest conditions in the present study are consistent with the interpretation that both distance and location were rehearsable, in that recall was not significantly affected by an unfilled retention interval. Unfortunately, universal agreement regarding the rehearsability of these cues has not been found (e.g., Diewert, 1975; Laabs, 1973). 
Added research is needed to determine the locus of stimulus selection and, in general, to determine the coordination of relevant encoding characteristics of simple motor movements. In the latter regard, Dickinson and Higgins (1977) have applied the traditional verbal proactive interference $(\mathrm{PI})$ paradigm to motor material. They examined the encoding of distance and direction cues; however, the same could be done for distance and location cues, as they have suggested. If distance and location can be dissociated during encoding, then PI release should result when the learning of distance is followed by a switch to location learning or vice versa.

Although the present results fit nicely within the framework of a specific-cue interpretation, caution must be exercised in extending this argument to studies that do not control learning strategies at original movement execution. When learning strategies are allowed to vary, multiple cues can be encoded (e.g., Gundry, 1975). In these situations, a multiple-cue hypothesis can account for interpolated movement effects (Hagman \& Williams, 1977). The present study demonstrates that students are able to apply a specificcue retention strategy when directed to by way of learning instructions.

Thus, it seems reasonable to conclude that motor movements may either be processed as unitary wholes comprised of multiple kinesthetic cues or they may be segmented into distinct, separate kinesthetic cues. When subjects are instructed to process movements in a segmental fashion, they tend to base their memorial representation of those movements on a single cue.

\section{REFERENCES}

ADAms, J. A. A closed-loop theory of motor learning. Journal of Motor Behavior, 1971, 3, 111-149.

Adams, J. A., \& Goetz, E. T. Feedback and practice as variables in error detection and correction. Journal of Motor Behavior, 1973, 5, 217.224.

Carmer, S. G., \& Swanson, M. R. An evaluation of ten pairwise multiple comparison procedures by Monte Carlo methods. Journal of the American Statistical Association, $1973,68,66.74$.

Craft, J. L., \& Hinrichs, J. V. Short-term retention of simple motor response: Similarity of prior and succeeding response. Journal of Experimental Psychology, 1971, 87, 297-302.
Dickinson, J., \& Higgins, N. Release from proactive and retroactive interference in motor short-term memory. Journal of Motor Behavior, 1977, 9, 61-66.

Diewert, G. L. Retention and coding in motor short-term memory: A comparison of storage codes for distance and location information. Journal of Motor Behavior, 1975, 7, 183-190.

Frost, N. H. Encoding and retrieval in a visual memory task. Journal of Experimental Psychology, 1972, 95, 317-326.

GUNDRY, J. The use of location and distance in reproducing different amplitudes of movement. Journal of Motor Behavior, 1975, 7, 91-100.

Hagman, J. D., \& Francis, E. W. The instructional variable and kinesthetic cue recall. Journal of Motor Behavior, 1975, 7, 141-146.

Hagman, J. D., \& Williams, E. Use of distance and location in short-term motor memory. Perceptual and Motor Skills, 1977, 44, 867-873.

LAABS, G. J. Retention characteristics of different reproduction cues in motor short-term memory. Journal of Experimental Psychology, 1973, 100, 168-177.

LAABS, G. J. The effect of interpolated motor activity on the short-term retention of movement distance and end-location. Journal of Motor Behavior, 1974, 6, 279-288.

PosNer, M. I. Coordination of internal cues. In W. G. Chase (Ed.), Visual information processing. New York: Academic Press, 1973.

Posner, M. I., Nissen, M. J., \& Klein, R. M. Visual dominance: An information-processing account of its origins and significance. Psychological Review, 1976, 83, 157-171.

Russell, D. G. Spatial location cues and movement reproduction. In G. E. Stelmach (Ed.), Motor control: Issues and trends. New York: Academic Press, 1976.

Stelmach, G. E. Kinesthetic recall and information reduction activity. Journal of Motor Behavior, 1970, 2, 183-194.

Stelmach, G. E. Feedback-a determiner of forgetting in short-term motor memory. Acta Psychologica, 1973, 37, 333-339.

Stelmach, G. E., \& Walsh, M. F. The temporal placement of interpolated movements in short-term motor memory. Journal of Motor Behavior, 1973, 5, 165-173.

UNDERWOOD, B. J. Stimulus selection in verbal learning. In C. N. Cofer \& B. S. Musgrave (Eds.), Verbal behavior and Learning: Problems and processes. New York: McGraw-Hill, 1963.

WrisberG, C. A. The serial-position effect in short-term motor retention. Journal of Motor Behavior, 1975, 7, 289-295.

ZAHORIK, D. M. Subject strategies and interference in a discrete motor task. Psychonomic Science, 1972, 28, 349-351.

(Received for publication November 28, 1977; accepted February 28,1978 .) 